\title{
Temperature dependence of the phase-coherence length in $\ln N$ nanowires
}

Ch. Blömers, Th. Schäpers, T. Richter, R. Calarco, H. Lüth, and M. Marso

Citation: Appl. Phys. Lett. 92, 132101 (2008);

View online: https://doi.org/10.1063/1.2905268

View Table of Contents: http://aip.scitation.org/toc/apl/92/13

Published by the American Institute of Physics

\section{Articles you may be interested in}

Universal conductance fluctuations and localization effects in $\ln N$ nanowires connected in parallel Journal of Applied Physics 108, 113704 (2010); 10.1063/1.3516216

Weak antilocalization and conductance fluctuation in a single crystalline Bi nanowire Applied Physics Letters 104, 043105 (2014); 10.1063/1.4863421

Phase coherence length of electron waves in narrow $\mathrm{AlGaAs} / \mathrm{GaAs}$ quantum wires fabricated by focused ion beam implantation

Applied Physics Letters 54, 2103 (1989); 10.1063/1.101177

Effect of Si-doping on InAs nanowire transport and morphology

Journal of Applied Physics 110, 053709 (2011); 10.1063/1.3631026

Weak localization and weak antilocalization in doped germanium epilayers

Applied Physics Letters 110, 062101 (2017); 10.1063/1.4975600

Long phase coherence length and anisotropic magnetoresistance in $\mathrm{MgZnO}$ thin film

Journal of Applied Physics 117, 155304 (2015); 10.1063/1.4918541

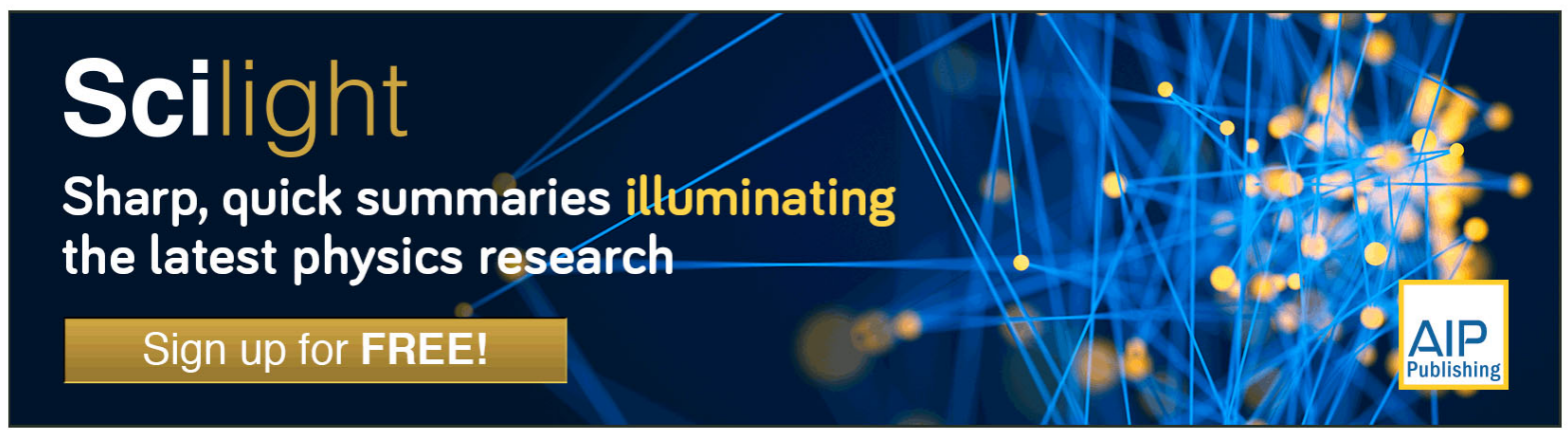




\title{
Temperature dependence of the phase-coherence length in InN nanowires
}

\author{
Ch. Blömers, Th. Schäpers, ${ }^{a}$ T. Richter, R. Calarco, H. Lüth, and M. Marso \\ Institute for Bio- and Nanosystems (IBN-1) and JARA Jülich-Aachen Research Alliance, Research Centre \\ Jülich GmbH, 52425 Jülich, Germany
}

(Received 5 February 2008; accepted 13 March 2008; published online 31 March 2008)

\begin{abstract}
We report on low-temperature magnetotransport measurements on InN nanowires, grown by plasma-assisted molecular beam epitaxy. The characteristic fluctuation pattern observed in the conductance was employed to obtain information on phase-coherent transport. By analyzing the root mean square and the correlation field of the conductance fluctuations at various temperatures, the phase-coherence length was determined. (c) 2008 American Institute of Physics.
\end{abstract}

[DOI: $10.1063 / 1.2905268$ ]

Semiconductor nanowires are versatile building blocks for the design of future electronic devices. ${ }^{1-4}$ This includes, e.g., nanoscaled transistors, ${ }^{5,6}$ resonant tunneling devices, ${ }^{7}$ or quantum dot based devices, ${ }^{8,9}$ to name just a few. Among the many possible materials suitable for semiconductor nanowires, InN is particularly interesting because of its low energy band gap and its high surface conductivity. ${ }^{10-13}$

At low temperatures, electron interference effects often play a prominent role in the transport characteristics of nanostructures. Typical phenomena observed in this regime are weak localization, the Aharonov-Bohm effect, or universal conductance fluctuations. ${ }^{14,15}$ The characteristic length connected to these effects is the phase-coherence length $l_{\phi}$, i.e., the length over which phase-coherent transport is maintained. The length $l_{\phi}$ is an important parameter for the design of device structures based on electron interference.

The analysis of conductance fluctuations is one of the possible methods, in order to obtain information on $l_{\phi}$ in semiconductor nanostructures. ${ }^{16-22}$ Due to the small dimensions of semiconductor nanowires, often only a limited number of scattering centers are involved in the transport. In this case, pronounced fluctuations in the conductance can be expected, e.g., when the magnet field is varied. This was indeed observed by Hansen et al. ${ }^{23}$ for InAs nanowires.

In this letter, we will exploit the characteristic fluctuation pattern in the magnetoresistance of $\mathrm{InN}$ nanowires, in order to obtain information on the phase-coherent transport. By analyzing the root mean square (rms) and the correlation field of the fluctuation pattern, the temperature dependence of $l_{\phi}$ will be determined.

Two InN nanowires of different thicknesses, prepared by plasma-assisted molecular beam epitaxy were investigated in this study. ${ }^{13}$ The wires were grown on a $\mathrm{Si}$ (111) substrate at a temperature of $475^{\circ} \mathrm{C}$ under N-rich conditions. For the growth of the first sample (wire A) a beam equivalent pressure for In of $3.0 \times 10^{-8}$ mbar was chosen, while for the second sample (wire B) $7.0 \times 10^{-8}$ mbar was adjusted. As illustrated in Fig. 1(a), using this scheme growth of $\mathrm{InN}$ nanowires with a length of approximately $1 \mu \mathrm{m}$ was achieved. From photoluminescence measurements, a typical overall electron concentration of $5 \times 10^{18} \mathrm{~cm}^{-3}$ was estimated. Details about the growth of the $\mathrm{InN}$ nanowires can be found in Refs. 12 and 13.

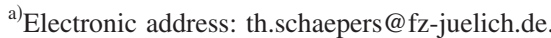

For the transport measurements of the InN wires, contact pads and adjustment markers were defined on a $\mathrm{SiO}_{2}$-covered $\mathrm{Si}$ (110) wafer. The $\mathrm{InN}$ nanowires were dispersed on the patterned substrate, by separating them first from their host substrate in an acetone solution. Subsequently, a droplet of acetone containing the detached $\mathrm{InN}$ nanowires was put on the patterned substrate wafer. In the final step, the wires were individually contacted using $\mathrm{Ti} / \mathrm{Au}$ electrodes defined by electron beam lithography. Wire A had a diameter of $d=67 \mathrm{~nm}$ with contact pads separated by $L$ $=410 \mathrm{~nm}$, while wire $B$ had a diameter of $130 \mathrm{~nm}$ with contacts separated by $530 \mathrm{~nm}$. A scanning electron micrograph of wire B is shown in Fig. 1.

The measurements were performed in a He- 3 cryostat at temperatures between 0.6 and $25 \mathrm{~K}$. The cryostat was equipped with a $10 \mathrm{~T}$ magnet. The magnetic field was oriented perpendicular to the axis of the wires. The magnetoresistance was measured by using a lock-in technique with an ac bias current of $30 \mathrm{nA}$.

The fluctuation pattern in the total magnetoresistance of wire A at various temperatures is shown in Fig. 2(a). The measurements were performed in a magnetic field range from -1.5 to $10.0 \mathrm{~T}$. It can be clearly seen that the fluctuation amplitude substantially decreases if the temperature is increased. Furthermore, one finds that although the amplitude is damped with increasing temperature, the pattern itself is reproduced. As can be seen in Fig. 2(a), due to the twoterminal measurement, the fluctuation pattern is symmetric with respect to the magnetic field $B$.

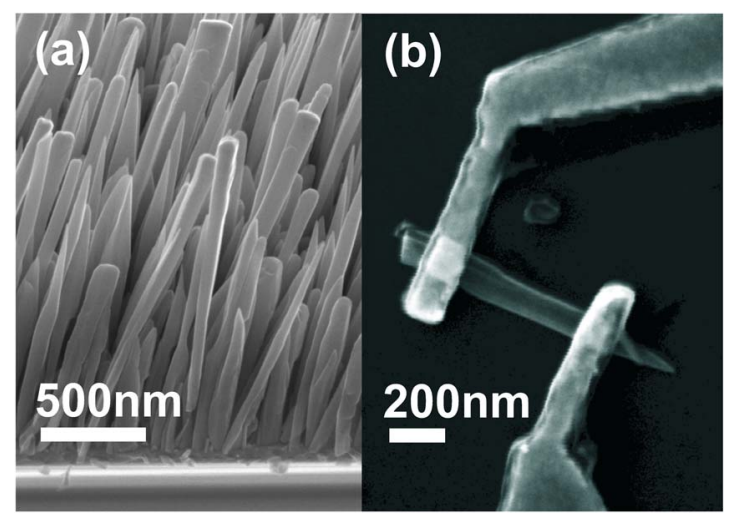

FIG. 1. (Color online) Scanning electron micrograph (a) of the as-grown InN nanowires used to prepare sample B and (b) of wire B with the individually defined $\mathrm{Ti} / \mathrm{Au}$ contact pads. 


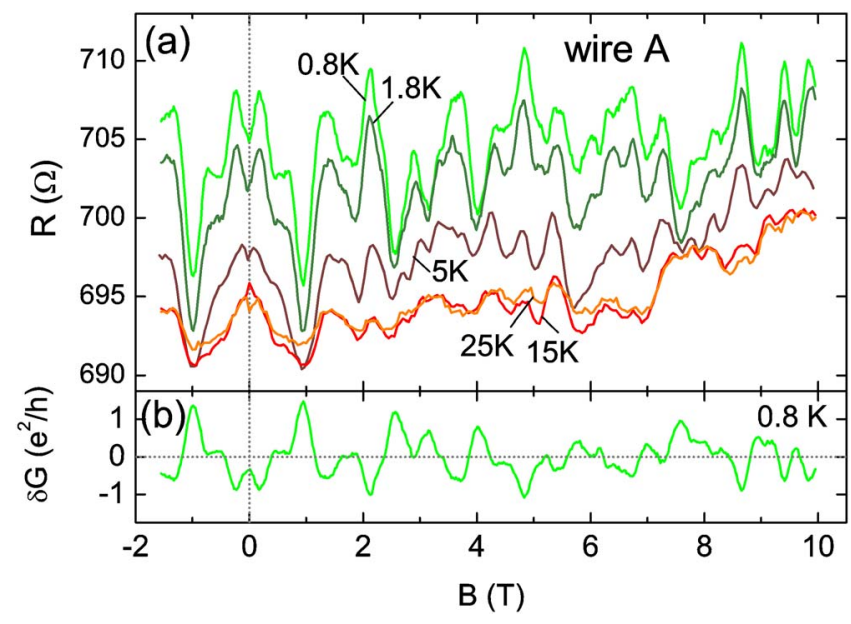

FIG. 2. (Color online) (a) Magnetoresistance of wire $A$ at temperatures between 0.8 and $25 \mathrm{~K}$. The measurements show the total two-terminal resistance including the contact resistance. (b) Normalized conductance fluctuations of wire $\mathrm{A}$ at $0.8 \mathrm{~K}$.

In order to analyze the electron interference phenomena in detail, first, the fluctuations in the magnetoresistance were converted to the corresponding conductance fluctuations $\delta G$. In Fig. 2(b), the fluctuation pattern of the normalized magnetoconductance $\delta G$ of wire $\mathrm{A}$ at $0.8 \mathrm{~K}$ is shown. Since the measurements were performed in a two-terminal configuration, first, the contact resistance $R_{c}$ was subtracted. The contact resistance was estimated by measuring a number of $\mathrm{InN}$ wires of the same growth run with comparable diameter but different contact separations. For wires A and B, contact resistances $R_{c}$ of $330 \pm 50$ and $250 \pm 50 \Omega$, were determined, respectively. After subtracting the contact resistance, the conductance fluctuations $\delta G=G-G_{0}$ were extracted by subtracting the slowly varying parabolic background contribution $G_{0}$ from the total wire conductance. As can be seen in Fig. 2(b), at $0.8 \mathrm{~K}$, the conductance fluctuation amplitude is in the order of $e^{2} / h$.

We will now focus on the analysis of the temperature dependence of the conductance fluctuations. The magnitude of $\delta G$ can be quantified by the rms of the conductance fluctuation $\operatorname{rms}(G)$, which is defined by $\sqrt{\left\langle\delta G^{2}\right\rangle}$. Here, $\langle\cdots\rangle$ represents the average over the magnetic field. In Fig. 3(a), the $\operatorname{rms}(G)$ of wire A is plotted as a function of temperature. Two regimes are revealed: at temperatures below about $1.5 \mathrm{~K}$, rms $(G)$ tends to saturate, whereas, at temperatures above $1.5 \mathrm{~K}$, a decrease of $\operatorname{rms}(G)$ proportional $T^{-0.4}$ is observed. A similar decrease proportional to $T^{-0.4}$ was found for wire $\mathrm{B}$ above $\approx 2 \mathrm{~K}$, while, below $2 \mathrm{~K}$, a slightly steeper decrease was observed compared to wire $\mathrm{A}$.

Before we proceed to discuss the temperature dependence of rms $(G)$, we will turn to another important quantity, the correlation field $B_{c}$. The correlation field is derived from the autocorrelation function of the conductance fluctuation which is defined as $F(\Delta B)=\langle\delta G(B+\Delta B) \delta G(B)\rangle{ }^{20}$ The full width at half maximum of the autocorrelation function $F\left(B_{c}\right)=\frac{1}{2} F(0)$ defines the correlation field $B_{c}$. In Fig. 3(b), the temperature dependence of $B_{c}$ is plotted for both wires. For wire $A$, the correlation field remains constant for temperatures smaller than $1 \mathrm{~K}$, while for larger temperatures, $B_{c}$ increases proportional to $T^{0.19}$. In contrast, for wire $\mathrm{B}$, the
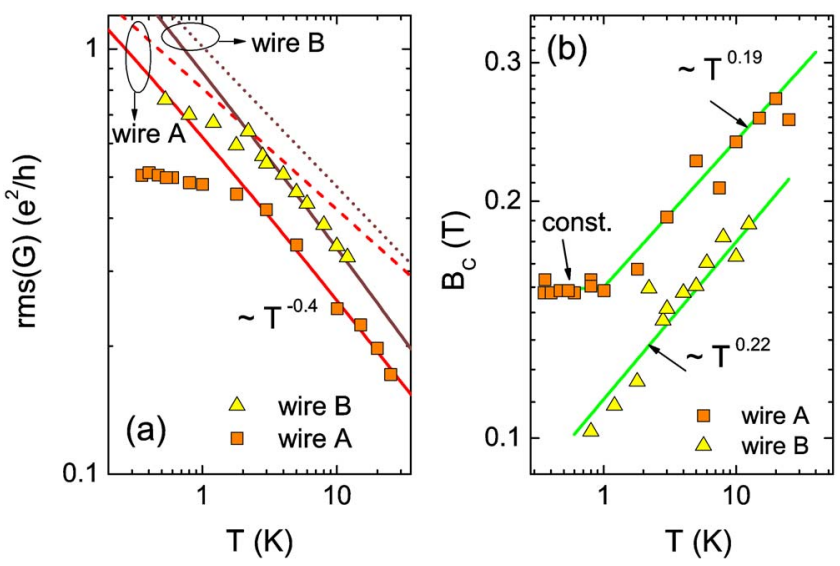

FIG. 3. (Color online) (a) Root mean square (rms) of the conductance fluctuations of wires A $(\square)$ and B $(\triangle)$ as a function of temperature. The full and dashed lines show the theoretically expected traces of rms $(G)$ including and excluding thermal averaging, respectively. (b) Correlation field $B_{c}$ vs temperature for wires $\mathrm{A}$ and $\mathrm{B}$. The full lines represent the exponential increase of $B_{c}$.

correlation field $B_{c}$ monotonically increases with $T^{0.22}$ in the whole temperature range.

In order to estimate the temperature dependence of $l_{\phi}$, we focus on the analysis of $B_{c}$. No attempt was made to determine $l_{\phi}$ directly from $\mathrm{rms}(G)$, because $\mathrm{rms}(G)$ depends on the interplay between two parameters: $l_{\phi}$ and $l_{T}{ }^{20}$ The thermal diffusion length $l_{T}=\sqrt{h \mathcal{D} / k_{B} T}$, with $\mathcal{D}$ the diffusion constant, is a measure for the thermal broadening. In contrast, $B_{C}$ does not depend on thermal broadening effects, i.e., on $l_{T}{ }^{22}$ Since, in our wires, the diameter $d$ exceeds the elastic mean free path $l_{e}, l_{\phi}$ was determined from $B_{c}$ using the expression for the diffusive regime: $l_{\phi} \approx \Phi_{0} / B_{c} d$, with $\Phi_{0}=h / e$ the magnetic flux quantum..$^{20,22}$ The results for sample A are shown in Fig. 4(a). For $T<1.5 \mathrm{~K}$, the calculated values of $l_{\phi}$ saturate at about the wire length $L$, indicating that phase coherence is maintained over the complete length. Above $1.5 \mathrm{~K}, l_{\phi}$ decreases with increasing temperature following a dependence proportional to $T^{-0.19}$. Using the same procedure as described above, $l_{\phi}$ was determined from $B_{c}$ for wire B, as well. As can be seen in Fig. 4(b), $l_{\phi}$ monotonously decreases with temperature following a depen-
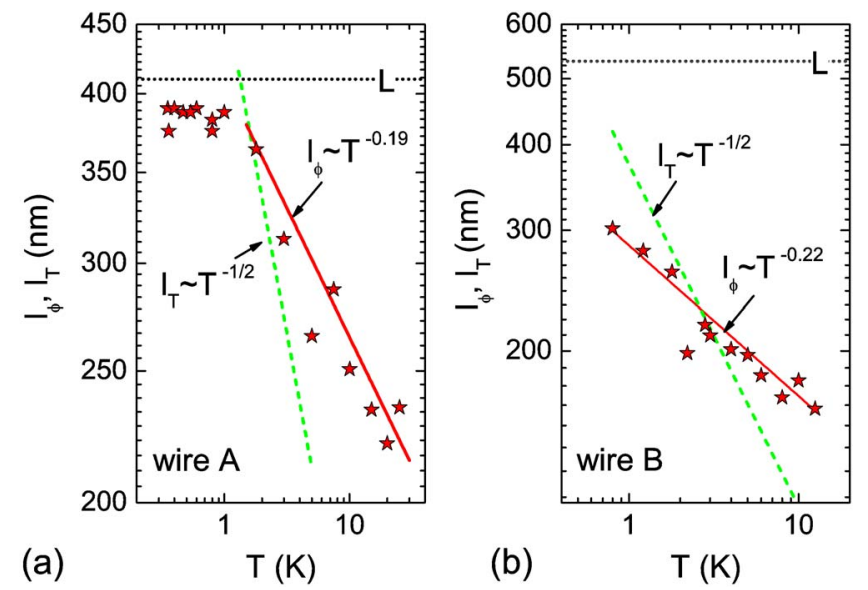

FIG. 4. (Color online) (a) Phase-coherence length $l_{\phi}$ of wire A determined from $B_{c}$ as a function of temperature $(\star)$. The solid line represents the $T^{-0.19}$ dependence of $l_{\phi}$ above $1.5 \mathrm{~K}$. The dashed line corresponds to the expected temperature dependence of $l_{T}$. The wire length $L$ is indicated by the dotted line. (b) Corresponding values for wire B. 
dence proportional to $T^{-0.22}$ in the whole temperature range. Consequently, one can state that, for this wire, $l_{\phi}$ is always smaller than $L$ in the temperature range considered here. For both wires, the temperature dependence of $l_{\phi}$ is slightly smaller than the theoretically expected dependence proportional to $T^{-1 / 3}$. $^{24}$ Presumably, the smaller phase-coherence length of wire B, being grown under different growth conditions than wire $\mathrm{A}$, is caused by a larger number of scattering centers.

Using the interpolation formula derived by Beenakker and van Houten: ${ }^{22}$

$$
\operatorname{rms}(G)=\alpha \frac{e^{2}}{h}\left(\frac{l_{\phi}}{L}\right)^{3 / 2}\left[1+\frac{9}{2 \pi}\left(\frac{l_{\phi}}{l_{T}}\right)\right]^{-1 / 2},
$$

the temperature dependence of $\operatorname{rms}(G)$ was estimated. The formula is valid for $l_{\phi} \approx l_{T}<L$. Ideally, the constant $\alpha$ has a value of $\sqrt{6}$. The calculated values for wires A and B using Eq. (1) are shown in Fig. 3. One finds that, at $T>1 \mathrm{~K}$, the experimental values of rms $(G)$ versus $T$ fit very well to the theoretical curves. The values of $\alpha$ with 0.38 and 1.32 for wires A and B, respectively, deviate to some extent from the theoretically expected value. This can be attributed to uncertainties in the determination of $R_{c}$ affecting the amplitude of rms $(G)$. For comparison, the rms $(G) \propto\left(l_{\phi} / L\right)^{3 / 2}$ curves, representing the case of neglected thermal averaging, are also shown in Fig. 3. One finds that, in this case, the decrease of rms $(G)$ is too small. The fact that thermal smearing has to be considered is also supported by comparing $l_{T}$ to $l_{\phi}$ (cf. Fig. 4). Here, one finds that $l_{\phi}$ and $l_{T}$ are in the same order of magnitude. At lower temperatures $(T<1 \mathrm{~K})$, the smaller slope of $\operatorname{rms}(G)$ can be explained by the reduced effect on $l_{T}$ and to the fact that $l_{\phi}$ exceeds or approaches $L$.

In summary, the phase-coherence length $l_{\phi}$ of InN nanowires was determined by analyzing the characteristic conductance fluctuation pattern. For the shorter wire, it is found that, at low temperatures, $l_{\phi}$ exceeds the wire length $L$, while at temperatures above $1.5 \mathrm{~K}, l_{\phi}<L$ and continuously decreases with increasing $T$. In contrast for the longer wire (wire B), $l_{\phi}$ was smaller than $L$ in the complete temperature range. Our investigations demonstrate that for short InN nanowires, phase-coherent transport can be maintained along the complete length.

${ }^{1}$ L. Samuelson, C. Thelander, M. T. Björk, M. Borgström, K. Deppert, K. A. Dick, A. E. Hansen, T. Martensson, N. Panev, A. I. Persson, W. Seifert, N. Sköld, M. W. Larsson, and L. R. Wallenberg, Physica E (Amsterdam) 25, 313 (2004).

${ }^{2}$ W. Lu and C. M. Lieber, J. Phys. D 39, R387 (2006).

${ }^{3}$ C. Thelander, P. Agarwal, S. Brongersma, J. Eymery, L. Feiner, A. Forchel, M. Scheffler, W. Riess, B. Ohlsson, U. Gösele, and L. Samuelson, Mater. Today 9, 28 (2006).

${ }^{4}$ K. Ikejiri, J. Noborisaka, S. Hara, J. Motohisa, and T. Fukui, J. Cryst. Growth 298, 616 (2007).

${ }^{5}$ T. Bryllert, L.-E. Wernersson, T. Lowgren, and L. Samuelson, Nanotechnology 17, 227 (2006).

${ }^{6}$ Y. Li, J. Xiang, F. Qian, S. Gradecak, Y. Wu, H. Yan, D. Blom, and C. M. Lieber, Nano Lett. 6, 1468 (2006).

${ }^{7}$ M. T. Björk, B. J. Ohlsson, C. Thelander, A. I. Persson, K. Deppert, L. R. Wallenberg, and L. Samuelson, Appl. Phys. Lett. 81, 4458 (2002).

${ }^{8}$ S. D. Franceschi, J. A. van Dam, E. P. A. M. Bakkers, L. Feiner, L. Gurevich, and L. P. Kouwenhoven, Appl. Phys. Lett. 83, 344 (2003).

${ }^{9}$ C. Fasth, A. Fuhrer, M. T. Bjork, and L. Samuelson, Nano Lett. 5, 1487 (2005).

${ }^{10}$ C. H. Liang, L. C. Chen, J. S. Hwang, K. H. Chen, Y. T. Hung, and Y. F. Chen, Appl. Phys. Lett. 81, 22 (2002).

${ }^{11}$ C.-Y. Chang, G.-C. Chi, W.-M. Wang, L.-C. Chen, K.-H. Chen, F. Ren, and S. J. Pearton, Appl. Phys. Lett. 87, 093112 (2005).

${ }^{12}$ T. Stoica, R. J. Meijers, R. Calarco, T. Richter, E. Sutter, and H. Lüth, Nano Lett. 6, 1541 (2006).

${ }^{13}$ R. Calarco and M. Marso, Appl. Phys. A: Mater. Sci. Process. 87, 499 (2007).

${ }^{14}$ C. W. J. Beenakker and H. van Houten, in Solid State Physics, edited by H. Ehrenreich and D. Turnbull (Academic, New York, 1991), Vol. 44, p. 1.

${ }^{15}$ J. J. Lin and J. P. Bird, J. Phys.: Condens. Matter 14, R501 (2002).

${ }^{16}$ C. P. Umbach, S. Washburn, R. B. Laibowitz, and R. A. Webb, Phys. Rev. B 30, 4048 (1984).

${ }^{17}$ A. D. Stone, Phys. Rev. Lett. 54, 2692 (1985).

${ }^{18}$ P. A. Lee and A. D. Stone, Phys. Rev. Lett. 55, 1622 (1985).

${ }^{19}$ B. Al'tshuler, Pis'ma Zh. Eksp. Teor. Fiz. 41, 530 (1985) [JETP Lett. 41, 648 (1985)].

${ }^{20}$ P. A. Lee, A. D. Stone, and H. Fukuyama, Phys. Rev. B 35, 1039 (1987).

${ }^{21}$ T. J. Thornton, M. Pepper, H. Ahmed, G. J. Davies, and D. Andrews, Phys. Rev. B 36, 4514 (1987).

${ }^{22}$ C. W. J. Beenakker and H. van Houten, Phys. Rev. B 37, 6544 (1988).

${ }^{23}$ A. E. Hansen, M. T. Börk, C. Fasth, C. Thelander, and L. Samuelson, Phys. Rev. B 71, 205328 (2005).

${ }^{24}$ B. L. Al'tshuler, A. G. Aronov, and D. E. Khmelnitsky, J. Phys. C 15, 7367 (1982). 